\title{
Modeling and Design of Digital Electronic Systems
}

\author{
Marcian Cirstea \\ Anglia Ruskin University, UK
}

\begin{abstract}
The paper is concerned with the modern methodologies for holistic modelling of electronic systems enabling system-on-chip design. The method deals with the functional modelling of complete electronic systems using the behavioral features of Hardware Description Languages or high level languages then targeting programmable devices - mainly Field Programmable Gate Arrays (FPGAs) - for the rapid prototyping of digital electronic controllers. This approach offers major advantages such as: a unique modelling and evaluation environment for complete power systems, the same environment is used for the rapid prototyping of the digital controller, fast design development, short time to market, a CAD platform independent model, reusability of the model/design, generation of valuable IP, high level hardware/software partitioning of the design is enabled, Concurrent Engineering basic rules (unique EDA environment and common design database) are fulfilled. The recent evolution of such design methodologies is marked through references to case studies of electronic system modelling, simulation, controller design and implementation. Pointers for future trends / evolution of electronic design strategies and tools are given.
\end{abstract}

Keywords-Design methodology, Computer aided engineering, Field programmable gate arrays, Digital integrated circuits, Digital systems, Artificial Intelligence

\section{INTRODUCTION}

The fast progress recently encountered in VLSI technology and Electronic Design Automation techniques has created the opportunity for the design and development of complex and high performance electronic systems targeting system-on-chip implementation. However, the creation of new technologies does not necessarily begin with new basic knowledge but with the innovative design, using existing knowledge, to meet a specific need and to achieve a desirable cluster of performance characteristics. Holistic modelling of complex electronic systems, using high level languages or hardware description languages, can constitute the first step towards novel designs. The outcome is a design environment allowing all system's parameters to be considered simultaneously at an early stage in the design process, therefore enabling an appropriate early hardware / software partitioning of the design along with functional simulation and thus maximizing performance.

Field Programmable Gate Arrays (FPGAs) have emerged in the late eighties and now constitute a mature technology. FPGAs are suited for high speed and generally demanding applications, with some of their design tools being very user friendly. Designers can develop a hardware architecture, dedicated to the implementation of a certain control algorithm
[1]. More complex devices available on the market also include DSP cores, rendering the FPGA as an ideal and effective target for electronic system-on-chip design implementation. The main limitation of this technology is still the cost, with another drawback being linked with the difficulty to integrate within current FPGAs mixed Analog / Digital peripherals like A/D and $\mathrm{D} / \mathrm{A}$ converters. However, this limitation was recently addressed with the introduction on the market of FPGAs which integrate $\mathrm{A} / \mathrm{D}$ converters.

The electronic systems holistic modelling and top-down design approach, initiated more than a decade ago [2], is correlated with a range of international initiatives [3] and leading edge research, directed towards systems modelling. The paper presents the recent developments related to such methodologies. The research carried out using such methods for electronic systems modelling, digital controller design and FPGA implementation is underpinned with examples.

\section{Holistic MODELING AND DESIGN APPROACH}

Traditionally, mathematical models have been developed to evaluate the functionality of global engineering systems. However, the practical development of each part of the system needs then to be separately addressed. This often used to involved other CAD tools and/or different software platforms, with the design being developed in a different environment.

Advances in CAD methodologies (Fig. 1) have brought the functional description of the design and practical hardware implementation closer, using hardware description languages (HDL), which are enabling the underpinning mathematical description and the electronic design implementation to be simultaneously addressed in a unique environment. Such languages (VHDL, Verilog) were successfully used for a few years now, synthesis tools having the ability to compile HDL designs into a range of target technologies, including FPGAs.

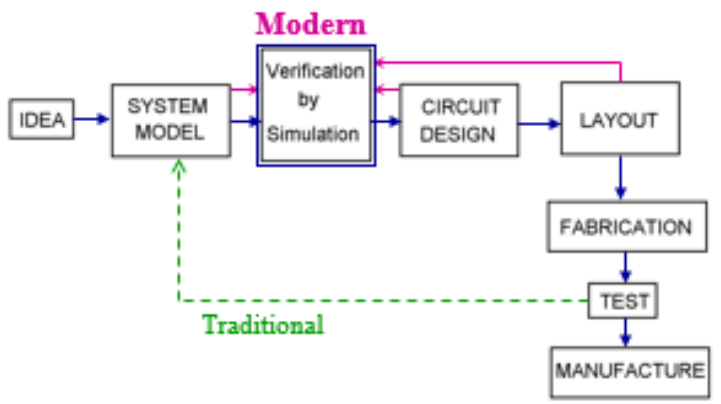

Fig. 1 Modern Electronic Systems Design Methodologies 


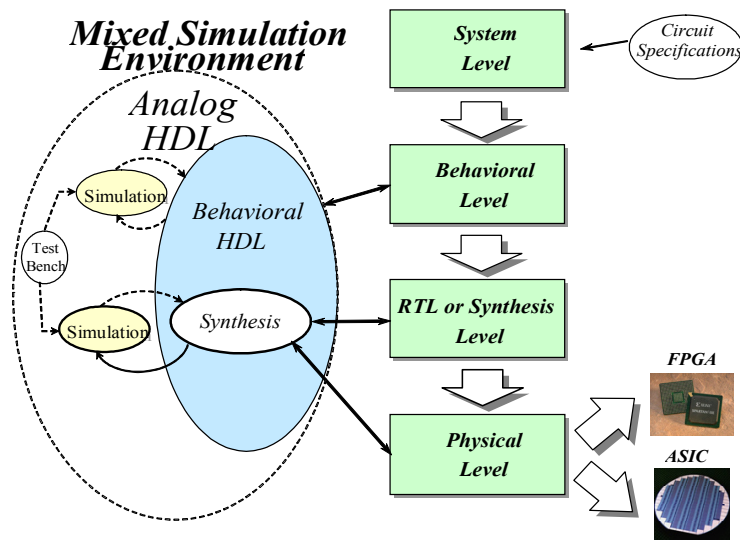

Fig. 2 Top-down electronic systems design approach

Thus, the behavioral description of an electronic system can be combined with a detailed electronic design, within rigorous implementation boundaries (Fig. 2). Electronic systems and a range of associated controllers can be modelled using this approach [4], [5], with benefits like:

- performance evaluation at an early stage of design

- functional modeling, controller design and hardware implementation performed on the same CAD platform

- system simulation as holistic model at all design stages

- Artificial Intelligence implementation is facilitated

- fast implementation and relatively short time to market

- versatile reusable design modules are obtained.

Simulation results are valuable to check circuit's behavior in its early stages of development but often it is the hardware validation that provides significant information before investing in a dedicated permanent chip configuration - ASIC. A fast and relatively cheap way to validate electronic system designs, enable by modern CAD tools, is via a prototype board containing re-programmable devices such as FPGAs [2], [5], [6]. The advantages of using this route are:

- ensures error-free design for permanent ASIC download

- shortens the time to correct any design problem.

- provides a common platform for investigating different control strategies / system topologies.

- Assures security / higher confidentially of the design

Modern complex FPGAs include microprocessor/DSP cores, resolving the dilemma (Fig. 3) of choosing the 'right' hardware target device [3] for an electronic controller implementation; the system-on-programmable-chip concept is nowadays fully accommodated in a single FPGA device. Thus, FPGAs have higher computational performance and lower power consumption than microprocessor based systems. Compared to ASIC implementation, FPGAs offer lower cost for initial deployment along with rapid deployment and configurability. Altogether, FPGAs thus constitute an ideal electronic systems-on-chip target device.

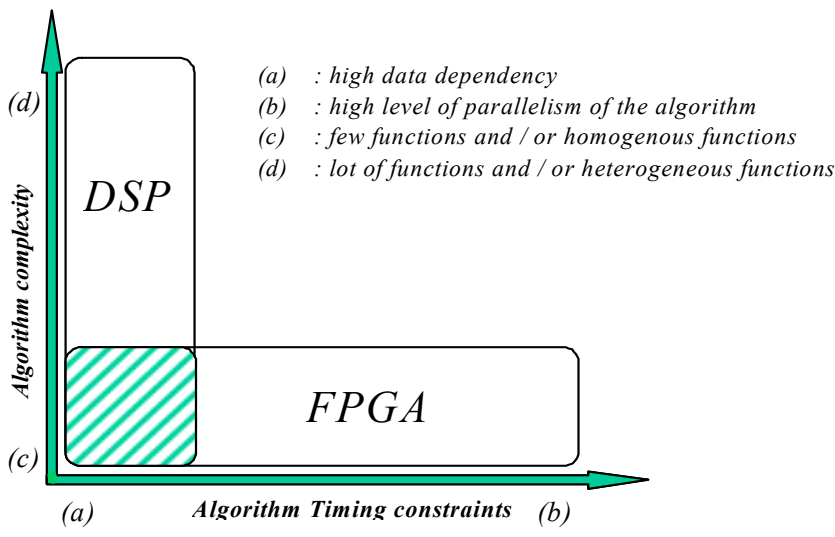

Fig. 3 Hardware implementation optimal target device selection

The general benefits of holistic modeling using high level languages (System-C, C-Synthesiser, Handle-C) or hardware description languages (VHDL, Verilog) combined with the advantages of modern FPGAs, enable novel complex but fast digital controllers to be modeled, simulated, hardware prototyped and evaluated with efficient use of resource.

\section{EARLy FPGA BASED ElECTRONIC CONTROL SYSTEMS}

\section{A. Case Study 1: FPGA Induction Motor Vector Controller}

Most a.c. drives traditionally adopted a microprocessor / DSP based digital control strategy, but implementation of the current loop and PWM control are still tied to analogue control circuitry. Although these control schemes have the advantage of fast dynamic response, they suffer the disadvantages of circuit complexity, limited functions and difficulties when circuit modification is required.

The reported work investigated the application of the novel approach, based on reusable VHDL digital architectures, to induction motor vector control modeling, simulation and FPGA rapid prototyping [7]. The universal applicability (reusability) of these control blocks to a wide range of vector control drives, with different topologies, is a major advantage. The employment of FPGAs for commissioning provides further benefits such as: rapid prototyping, simple design and compact implementation [8].

\section{B. Case study 2: ANN FPGA Induction Motor Controller}

An algorithm for ANN hardware implementation using a combination of AND gates, OR gates and Threshold Gates (TG), leads to compact hardware structures but it cannot be used for direct FPGA implementation because TGs are not available in FPGA logic cells [9]. In order to minimize both ASIC and FPGA hardware implementation of ANNs composed of neurons with step activation functions (but the method can be extended to sigmoidal functions), the solution adopted [10] was to treat each neuron as a Boolean function, implementing it separately, thus enabling the implementation complexity minimization. The proposed algorithm generates a multilayer pyramidal hardware structure, where layers of AND gates alternate with layers of OR gates. The bottom layer consists of incomplete NOT gates, a structure to be optimized later by 


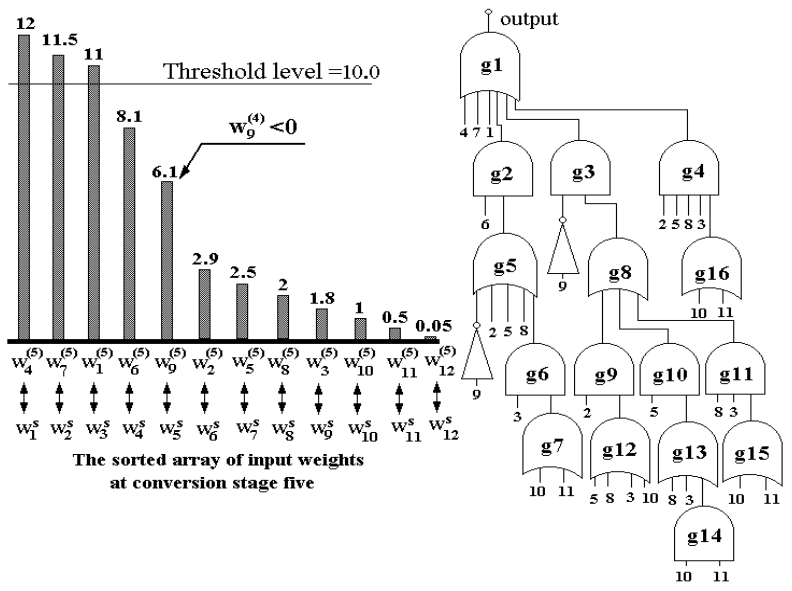

Fig. 4 Digital mathematical model to gate conversion

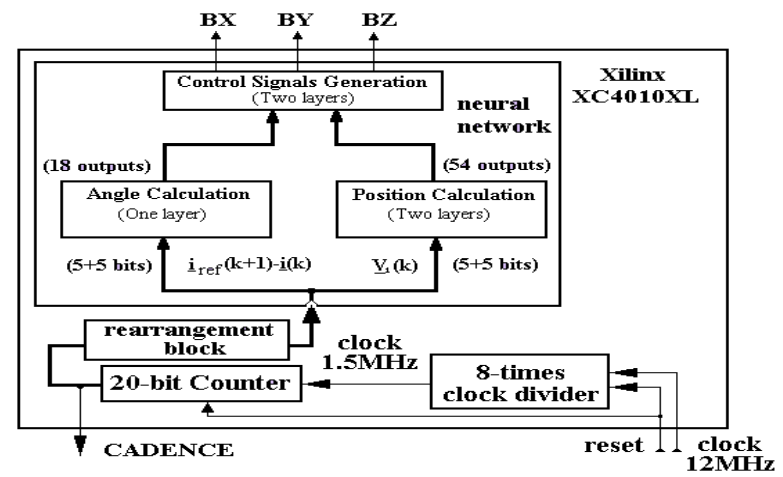

Fig. 5 ANN structure and testbench for operation speed testing

eliminating redundant logic gates groups. However, the method is effective only when the numbers of inputs and bits on each input are low, otherwise a classical circuit is more efficient.

The algorithm itself contains three steps: i) ANN mathematical model digitization, ii) conversion of the digitized model into a logic gate structure, and iii) hardware optimization by elimination of redundant logic gates. A set of $\mathrm{C}++$ programs automates algorithm implementation, generating optimized VHDL code. The sample in Fig. 4 shows a neuron with 12 input weights and positive threshold level. The weights are sorted in descending order and a recursive implementation starts [5], leading to the design of a feed forward ANN with 3 subnetworks (Fig. 5), generating the PWM switching pattern for an inverter. This circuit is part of an induction motor controller implemented in Xilinx XC4010XL FPGA.

\section{Case study 3: FPGA Fuzzy Logic Controller for Diesel Driven Synchronous Generators}

This example describes the design of an electronic control system allowing variable speed operation of diesel-driven stand-alone synchronous generators [4]. A control scheme that can isolate the final output frequency of the system from the effects of speed variations is simulated and designed (Fig. 6). The proposed design aims to improve the efficiency of diesel driven generators by allowing optimum speed operation.

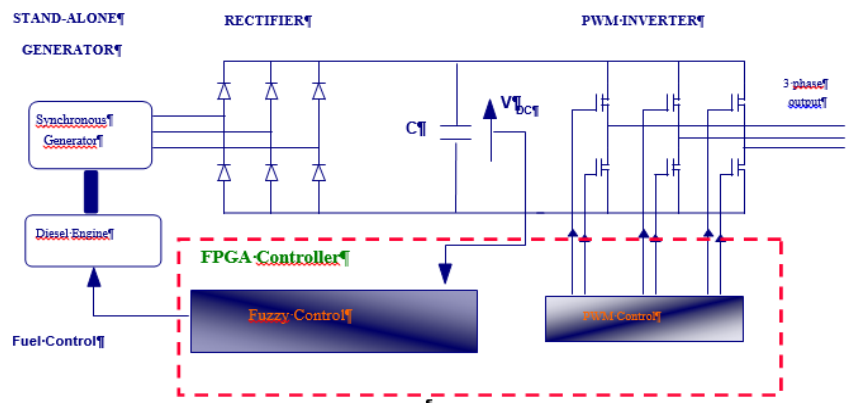

Fig. 6 Fuzzy Logic Controlled power system

A d.c. link power converter is used, the Pulse Width Modulation (PWM) inverter stage supplying the load. This configuration is widely used in variable speed wind energy conversion systems [11], [12]. A suitable fuzzy logic control system is designed to control the fuel valve of the diesel engine based on the d.c. link voltage input. The global control function ensures that the output voltage of the system maintains the desired magnitude and frequency over a range of varying rotational speed and loading conditions. The complete system was modeled and simulated using VHDL [4] and then the controller design was synthesised and prototyped into a Xilinx XC4010 FPGA. By adjusting the speed of the engine to the operating conditions, fuel consumption can be reduced while the same torque is produced. The main achievements are:

- the final output voltage is independent of the generator speed, allowing efficient speed operation at all times.

- the controller maintains the output voltage at the desired magnitude and frequency against changes in $\mathrm{V}_{\mathrm{dc}}$ which arise from changes in speed and/or load.

- the system provides a suitable platform for the study of efficient diesel engine driven variable speed generators.

\section{More Recent Artificial InTELligence BASED ELECTRONIC CONTROLLERS TARGETING FPGA}

The use of modern Electronic Design Automation packages for electronic systems design facilitates easy implementation of complex control algorithms and Artificial Intelligence (AI) into hardware. Hence, a range of complex and intelligent controller designs with industrial applications has been developed ([13][17]). Many of them target FPGAs, due to their rapid prototyping features and flexibility, especially through the recent availability of microprocessor or DSP cores, allowing hardware software co-design and implementation [18].

\section{A. Artificial Neural Network Based Control Systems}

FPGAs have been successfully used to implement Artificial Neural Networks (ANNs). Publications like [19], [20], [21] consider the FPGA as an effective implementation solution of control algorithms for industrial applications. Hardware implemented ANNs have an important advantage over computer simulated ones by fully exploiting the parallel operation of the neurons, thus achieving high information processing speed. An European Network of Excellence (ENE) report [22] indicates that the future implementation of 
hardware neural networks is shaped in 3 ways: i) by developing advanced techniques for mapping neural networks onto FPGA, ii) by developing innovative learning algorithms which are hardware-realizable, iii) by defining high-level descriptions of the neural algorithms in an industry standard to allow full simulations and fabrication and to produce demonstrators of the technology for industry. Such new designs will be of use to industry if the cost of adopting them is sufficiently low. Hardware-based neural networks are important to industry as they offer low power consumption and small size compared to PC software and they can be embedded in a wide range of systems. Matlab software libraries exist for traditional ANN models; the industry-standard format is however Verilog/VHDL or $\mathrm{C}++$ parameterized modular code, allowing customization.

An interesting trend is given by the recent development and implementation results of a parameterized FPGA-based architecture for feed-forward multi-layer perceptrons with back-propagation learning algorithm (FF-MLPs) presented in [23]. The proposed architecture makes possible native prototyping and design space exploration in hardware. Others papers exploit the learning ability of ANNs. Paper [24] presents an adaptive low-speed-damping controller for a stepper motor which removes nonlinear disturbance at low speeds, whereas paper [25] shows a self-tuning PID FPGAbased motion controller using RBF NN for an X-Y table.

\section{B. Fuzzy Logic Based Control Systems}

Today, fuzzy logic based control systems, or simply, Fuzzy Logic Controllers (FLCs) can be found in a growing number of products (washing machines, autofocus cameras, boats) as well as in more complex systems, such as smart grid controllers. Control engineers used to rely on mathematical models for their designs but the more complex a system is, the less effective the mathematical model. The success of fuzzy logic controllers is mainly due to their ability to cope with knowledge represented in a linguistic form instead of representation in the conventional mathematical framework.

FPGAs constitute an appropriate target for the implementation of fuzzy-logic controllers, facilitated by the flexibility of the design environment, enabling direct implementation of the circuit's abstract model. A high number of works have been published on fuzzy logic-based control systems. One paper presents a method employing hardware/software co-design techniques according to a preestablished partition of the tasks assigned to the selected components. This makes possible to tackle the controller prototyping as one of the design stages. In this case, the platform considered for prototyping has been a development board containing a standard microcontroller and an FPGA. Experimental results validate the approach [26].

A paper presents a novel approach to implement the fuzzy logic controller for speed control of electric vehicle by using FPGAs [27], whereas another paper [28] describes an implementation of a FLC on a reconfigurable FPGA system. An H-bridge multilevel converter governed by an integrated fuzzy logic controller / modulator designed with VHDL and implemented in FPGA is described in [29].
A design environment for the synthesis of embedded FLC on FPGAs, which provides a novel implementation technique, has been developed [30], which allows accelerating the exploration of the design space of fuzzy control modules, as well as a co-design flow that eases their integration into complex control systems.

The examples listed in this section demonstrate the good match between ANN and Fuzzy-Logic on one side and FPGAs on the other side, allowing a confident estimation of the increase use of FPGAs for artificial intelligence based digital electronic controllers' implementation.

\section{Digital Systems Design - Modern TREnds}

\section{A. General context - Integration and Globalization}

The last quarter of century has known an unprecedented evolution of the computational capacity, both in terms of hardware and software, alongside the apparition and exponential growth of the internet and the integration scale of silicon devices targeted for electronic circuit hardware implementation (following Moore's Law). In parallel, but also in close interconnection, an evolution of the computer aided engineering design tools has taken place. These have evolved from 'islands of automation' to completely integrated design packages, able to handle high levels of complexity and abstraction. The keywords characterizing this recent evolution of computational capacity, electronic devices, internet and design tools, all taken together, could be Integration and Globalization, leading to a significant change in the electronic systems' design methodology. The emerging modern approach has led to new generations of products surrounding us nowadays as the most natural companions in our lives (smart mobile phones, tablets, mini-computers, smart TVs, etc.). These can only be achieved via automated design and manufacturing methods, enabling their holistic modelling and functional optimization in a top-down approach.

To summarize such evolution in an easy to remember word, well characterizing over two decades of progress in the world of electronics and associated design tools and techniques, we can think of the word DIGIT. The letters can be taken as an acronym, to mean: Down (traditional electronic circuit design approaches being Down-up, starting at the bottom of an imaginary pyramid of building blocks and leading to the top - a system), Informatics (referring to the progressively increased capacity and use of computational power and the internet), Globalization, Integration and Top (referring to the modern top-down design methodologies, making use of higher level of abstraction in design specification and testing).

Taking a step backwards, we can 'validate' this trend as affecting our everyday life experiences as well. If we consider food shopping, to take just one example, nowadays we rarely approach this in discrete bits, at the bottom level of an imaginary pyramid, by planning to buy bread today, milk tomorrow, and then eggs the following day. The problem is rather approached by considering it holistically and resolved via a supermarket, where we buy everything we need in one go (optimizing our solution to the problem) and in many cases by internet order. So, the approach is top-down. 


\section{B. Integrated engineering modeling and design environment}

A range of early initiatives/developments aimed to achieve an overall engineering systems modeling environment at higher level of abstraction, enabling a test environment to be created early in the design cycle. This would allow the holistic evaluation of engineering systems with optimized integrated functionality, further enabling the more detailed design and development of its separate components. Just a few initiatives will be mentioned here.

Rosetta was an IEEE initiative (1999-2000) of system-level design language that allows users to create reusable components that specify the structure and behaviors of their system and subsystems. It can be used for modeling / describing engineering systems, combining complex electronic systems with complex mechanical systems. It consists of syntax (set of legal descriptions) and semantics (meaning associated with each description).

The Millenium Machine was a pioneering initiative (2000) of UK's Engineering and Physical Sciences Research Council (EPSRC) for holistic modeling of engineering systems (systems of systems). The aim was to achieve a fully digital model of an engineering system (an aircraft, for example).

The more recent Modelica is an object-oriented equation based modeling language for complex physical systems containing mechanical, electrical, electronic, hydraulic, thermal, control, electric power or process-oriented subcomponents. It allows, for example, the graphical modeling of electronics (Bond Graphs, linked to SPICE) or mechanical parts (Dymola, which offers animated models for CATIA).

The examples above illustrate the modern trend towards integration of computer aided design tools and the evolution towards higher level of abstraction in order to address topdown the complex functionality of electronic systems and to achieve combined optimization of their subsystems.

\section{Digital hardware designed using software engineering}

For electronic systems in particular, a range of languages and hardware design environments were recently developed, in an overall aim to increase the abstraction level of electronic system modelling, simulation and design methodologies in a top-down electronic system-on-chip design approach. An early development of such language was Handel-C - a compiler for Hardware-Software co-design from Celoxica (then Agilent Technology, then Mentor Graphics, 1995-2000). Matlab and Simulink, tools used on a very wide scale in electronic systems mathematical modelling, had developed a range of dedicated toolboxes, thus streamlining the rapid hardware prototyping of digital electronic control systems associated with the overall electronic, electrical or mechatronic system modelled, bridging the gap from mathematical modelling tools and hardware prototyping techniques such as those based on DSP / microprocessor compilers or hardware description languages facilitating FPGA hardware implementation.

Due to the availability of multi-million-gate (silicon) chips in the form of reconfigurable hardware (such as FPGAs, which also contain DSP/microprocessor cores), design methods had to change to enable faster time-to-market for complex products.
Hardware Description Languages' flexibility and choice of modelling styles are not anymore sufficient and they don't allow a 'fast enough' product development and market launch.

C-Synthesizer and System-C are more current / modern electronic circuits design environments / compilers, allowing high level language modeling / simulation / design of hardware integrated circuits. These enable software engineering methodologies to be deployed as the more effective modern solution for faster, compact and energy efficient hardware design in the form of embedded electronic systems-on-chip. Thus, modern CAD environments for electronics enable software engineers to design hardware.

\section{CONCLUSIONS}

Holistic modeling and digital electronic controller design techniques for the integrated development of industrial electronic systems are discussed. These are based on high level languages or hardware description languages and allow rapid FPGA prototyping of their electronic controllers as system-onchip. The electronic controllers are developed from idea, through the design and simulation stages, to complete systems, in a short time, giving further advantages such as high degree of flexibility, a reliable framework for design verification and high confidence in the correct first time circuit operation. The approach also provides a range of choices for the implementation target technology and wide compatibility of the design with respect to multiple existing modern CAD tools. This allows the integration of electronic controller models in complex electronic system models.

There is a wide range of applications for FPGA controlled electronic circuits and systems in automation, robots, electric drives, power electronics and generator systems ([31], [32], [33], [34]). It can be estimated that holistic modeling methodologies based on high level or hardware description languages, employing advanced $\mathrm{CAD}$ tools for hardware implementation of the digital controllers as system-on-chip will be increasingly used in the near future, the new trend being the use of software engineering methods and software specialists to develop / design hardware electronic circuits as system-on-chip solutions.

\section{REFERENCES}

[1] A. Nelson, T. Marcelo, "Custom Architectures for Fuzzy and Neural Networks Controllers,” JCS\&T, vol.2, no.7, pp. 9-15, 2002.

[2] M.N. Cirstea, A. Dinu, J. Khor, M. McCormick, "Neural and Fuzzy Logic Control of Drives and Power Systems", Elsevier Science, Oxford, 2002.

[3] E. Monmasson, E., L. Idkhajine, M.N. Cirstea, L. Bahri, A. Tisan, W. Naouar, "FPGAs in Industrial Control Applications", IEEE Trans. on Ind. Informatics, vol. 7, no. 2, pp. 224-244, 2011.

[4] J. Khor, "Intelligent Fuzzy Logic Control of Generators", PhD Thesis, De Montfort Univ., UK, 1999.

[5] A. Dinu, "FPGA Neural Controller for Three Phase Sensorless Induction Motor Drive Systems", PhD Thesis, De Montfort University, UK, 2000.

[6] Y.Y. Tzou, H.J. Hsu: "FPGA Realization of Space-Vector PWM Control IC for Three-Phase PWM Inverters". IEEE Trans. on Power Electronics, vol. 12, No. 6, November 1997, pp. 953-963. 
[7] A. Aounis: "An Investigation into Induction Motor Vector Control Based on Reusable VHDL Digital Architectures and FPGA Rapid Prototyping", PhD Thesis, De Montfort University, UK, 2002.

[8] A. Aounis, M. McCormick, M.N. Cirstea: "A Novel Approach to Induction Motor Controller Design and Implementation", Proc. of IEEE Power Conversion Conference (PCC), Osaka, pp.993-998, April 2002.

[9] A. Dinu, M. N. Cirstea: "A Digital Neural Network FPGA Direct Hardware Implementation Algorithm”, Proc. of ISIE 2007, Vigo, Spain, pp.2307-2312.

[10] A. Dinu, M.N. Cirstea, S.E. Cirstea: "Direct Neural Networks Hardware Implementation Algorithm", IEEE Trans. on Ind. Elec., vol. 57, no. 5, pp.1845-1848, 2010.

[11] A.M. Hilloowala, Sharaf: "A Rule Based Fuzzy Logic Controller for a PWM Inverter in a Stand Alone Wind Energy Conversion Scheme," IEEE Trans. on Ind. Apps., vol.32, no.1, pp.57-65, 1996.

[12] Simoes, et. al., "Design and Performance Evaluation of a Fuzzy-LogicBased Variable Speed Wind Generation System" IEEE Trans. on Inds. Apps., vol. 33, no. 4, pp. 956-965, 1997.

[13] O. López, J. Alvarez, J. Doval-Gandoy, et. al., "Comparison of the FPGA implementation of two multilevel space vector PWM algorithms" IEEE Trans. on Ind. Electronics, vol.55, no. 4, pp. 1537-1547, 2008.

[14] Da Zhang, Hui Li, "A Stochastic-Based FPGA Controller for an Induction Motor Drive With Integrated Neural Network Algorithms," IEEE Trans. on Ind. Electronics, vol. 55, no. 2, pp. 551-561, Feb. 2008.

[15] B. K. Bose, "Neural Network Applications in Power Electronics and Motor Drives - An Introduction and Perspective", IEEE Trans. on Ind. Electronics, vol. 54, no. 1, pp. 14-33, Feb. 2007.

[16] X. Shao, D. Sun, "Development of a New Robot Controller Architecture with FPGA Based IC Design for Improved High-Speed Performance", IEEE Trans. Ind. Informatics, vol. 3, no. 4, pp 312-321, 2007.

[17] Pedro Martín, Emilio Bueno et al., "A Methodology for Optimizing the FPGA Implementation of Industrial Control Systems", IECON '09. pp. 2831-2836, 2009.

[18] E. J. Bueno, Á. Hernández, et al., "A DSP and FPGA-based industrial control with high speed communication interfaces for grid converters applied to distributed power generation systems", IEEE Trans. on Industrial Electronics, vol. 56, no. 3, pp. 654-6691. March 2009.

[19] B. M. Wilamowski, H. Yu, "Improved computation for LevenbergMarquardt training”, IEEE Trans Neural Netw., 21(6), pp .930-937, 2010.

[20] B. Vasumathi, S. Moorthi, "Implementation of hybrid ANN-PSO algorithm on FPGA for harmonic estimation", Engineering Applications of Artificial Intelligence, vol. 25, no.3, 2012, pp. 476-483, Elsevier.
[21] M. Pietras, "Hardware conversion of neural networks simulation models for neural processing accelerator implemented as FPGA-based SoC", Int. Conf. on Field Programmable Logic and Appl. (FPL), 2014, pp.1-4.

[22] NEuroNet Roadmap. Future Prospects for Neural Networks, 2001. http://www.eunite.org/eunite/roadmap/02Roadmap1.pdf

[23] A. Gomperts, A. Ukil, F. Zurfluh, "Development and Implementation of Parameterized FPGA-Based General Purpose Neural Networks for Online Applications", IEEE Trans. on Ind. Inform., vol.7, no.1, pp.7889, 2011.

[24] Q. N. Le, J.W. Jeon, "Neural-Network-Based Low-Speed-Damping Controller for Stepper Motor with an FPGA", IEEE Trans. on Ind. Electronics, Vol. 57, Issue 9, pp. 3167-3180, September 2010.

[25] Ying-Shieh Kung, Ming-Shyan Wang, Tzu-Yao Chuang, "FPGA-based self-tuning PID controller using RBF neural network and its application in X-Y table", IEEE Int. Symposium on Industrial Electronics ISIE, pp. $694-699$, July 2009.

[26] S. Sanchez-Solano, R. Senhadji, A. Cabrera, I. Baturone, C. J. Jimenez A. Barriga, "Prototyping of Fuzzy Logic-Based Controllers Using Standard FPGA Development Boards", Proc. IEEE Int. Workshop on Rapid System Prototyping (RSP'02), 2002, CD-ROM.

[27] S.Poorani, T.V.S. Urmila Priya, K. Udaya Kumar, S. Renganarayanan: "FPGA Based Fuzzy Logic Controller For Electric Vehicle", Journal of the Institution of Electrical Engineers, Singapore, vol. 45, no. 5, 2005.

[28] D. Kim: "An implementation of fuzzy logic controller on the reconfigurable FPGA system," IEEE Trans. on Industrial Electronics, Vol. 47, no. 3, pp.703-715, 2000.

[29] C. Cecati, F. Ciancetta, P. Siano, "A FPGA/fuzzy logic - Based multilevel inverter", IEEE Int. Symposium on Industrial Electronics ISIE, pp. 706-711. July 2009.

[30] Juang Chia-Feng, Lu Chun-Ming, Lo Chiang, and Wang Chi-Yen, “Ant Colony Optimization Algorithm for Fuzzy Controller Design and its FPGA Implementation", IEEE Trans. on Ind. Electronics, vol.55, no.3, pp. 1453-1462, March 2008.

[31] B.K Bose, "Power Electronics And Motor Drives: Advances and Trends", Prentice Hall, 2006

[32] M. Wilamowski, J.D. Irwin, "Power Electronics and Motor Drives (Elect. Engineering Handbook)", CRC Press, 2011.

[33] T. Vetter, M. Schulz, "FPGA to Control Power Electronics", Power Electronics Europe, Issue 6, 2014, pp.19-21.

[34] M. Di Paolo Emilio, "Embedded Systems Design for High-Speed Data Acquisition and Control", Springer, 2015. 